46 | 2011

Accueil et formation des enfants étrangers en France de la fin du XIX $X^{e}$ siècle au début de la Deuxième Guerre mondiale

\title{
L'exception polonaise
}

Janine Ponty

\section{(2) OpenEdition}

Journals

Édition électronique

URL : https://journals.openedition.org/dhfles/2083

DOI : $10.4000 /$ dhfles.2083

ISSN : 2221-4038

Éditeur

Société Internationale pour l'Histoire du Français Langue Étrangère ou Seconde

Édition imprimée

Date de publication : 1 janvier 2011

Pagination : $33-45$

ISSN : 0992-7654

Référence électronique

Janine Ponty, "L'exception polonaise », Documents pour l'histoire du français langue étrangère ou seconde [En ligne], 46 | 2011, mis en ligne le 08 septembre 2014, consulté le 31 mai 2021. URL : http:// journals.openedition.org/dhfles/2083 ; DOI : https://doi.org/10.4000/dhfles.2083

Ce document a été généré automatiquement le 31 mai 2021

(c) SIHFLES 


\title{
L'exception polonaise
}

\author{
Janine Ponty
}

1 L'importance de la question scolaire au sein de l'immigration polonaise était telle dans l'entre-deux-guerres, que j'y avais consacré l'un de mes premiers articles, paru en 1977 dans la revue Relations Internationales. Et je l'avais intitulé « Les problèmes soulevés par la scolarisation des enfants polonais en France après la Première Guerre mondiale ${ }^{1}$.

2 Point commun avec la situation des Belges en France: dans les deux cas, nous observons une concentration particulière en des lieux donnés. Si Roubaix compte plus de Belges que de Français au recensement de 1881, les cités minières construites autour des puits de charbon, de fer ou de potasse assistent au cours des années 1920 à l'arrivée massive d'ouvriers polonais accompagnés ou vite rejoints par leurs femmes et leurs enfants. Prenons l'exemple de Sallaumines (Pas-de-Calais). Trois des fosses de la compagnie des mines de Courrières, les $n^{\circ} 4, n^{\circ} 5$ et $n^{\circ} 13$ se trouvent sur son territoire. Endeuillée le 10 mars 1906 par la catastrophe de Courrières, évacuée et détruite pendant la Grande Guerre, la localité ne compte que 1800 habitants en 1919. En partie grâce au retour de mineurs français, en partie sous l'effet de l'immigration, elle monte d'un bond à 14000 en 1926 et plafonne à ce niveau jusqu'à la crise économique des années trente. Les premiers Polonais arrivèrent en 1922. Très vite, ils approchèrent de la moitié de la population et, en 1931, dépassent en nombre les autochtones : les 14749 personnes recensées se répartissent alors entre 6801 Polonais, 6379 Français et 1569 autres étrangers (Tchécoslovaques, Hongrois, Yougoslaves, Italiens, Belges) ${ }^{2}$.

3 À cette date, les Polonais sont devenus la deuxième nationalité étrangère en France derrière les Italiens : 507800 Polonais, 808000 Transalpins. Les Espagnols pointent à la troisième place avec 352000 âmes et les Belges à la quatrième (254 000), eux qui, au XIX ${ }^{e}$ siècle s'affichèrent si longtemps en tête du classement.

\section{La volonté d'un enseignement dans la langue d'origine}

Contrairement à l'intelligentsia polonaise au sein de laquelle il est alors d'usage de parler français, les nouveaux arrivants viennent surtout de la campagne et, en règle générale, n'ont été que peu scolarisés. Ce qui s'explique aisément. En 1919, la Pologne 
sort de plus d'un siècle d'effacement étatique, pays confisqué au profit de trois puissances copartageantes (Russie, Prusse, Autriche). Or l'école n'était obligatoire ni dans l'empire des tsars, ni dans celui des Habsbourg. Seule la Prusse (devenue l'Allemagne en 1871) l'avait instituée. Les Polonais qui ont fréquenté l'école allemande lisent et écrivent en allemand. Tous, quelle que soit leur région d'origine, ont des notions orales d'allemand ou de russe. Mais ils n'ont jamais entendu un mot de français, ce qui contribue à leur dépaysement et aux difficultés relationnelles avec leur nouvel entourage.

5 Les Polonais possèdent une longue tradition migratoire. À la fin du XIX ${ }^{e}$ siècle, plus de deux millions d'entre eux avaient débarqué aux États-Unis. Or partout où ils s'installaient, ils reconstituaient un milieu national, généralement centré autour d'une église et d'une école : messes en polonais dites par un prêtre polonais, enseignement en polonais. L'exemple le plus connu est celui de Chicago grâce aux travaux de la célèbre école de sociologie de Chicago qui, la première, s'est penchée sur le problème du déracinement des immigrés et de leurs difficultés d'adaptation ${ }^{3}$. D'autres chercheurs, depuis, ont affiné leurs propos mais sans contredire leurs thèses. Il existe indéniablement chez les Polonais une symbiose entre le sentiment national et le sentiment religieux : au XIX siècle, en l'absence d'un État indépendant, ce sont les prêtres qui ont entretenu la flamme patriotique, luttant contre les effets de la russification ou de la germanisation, enseignant le catéchisme en polonais, faisant circuler sous le manteau les livres interdits. À la fin de la messe, tant en Pologne qu'à l'étranger et ceci jusqu'à nos jours, les fidèles chantent Boże Coś PolskĘ (Dieu sauve la Pologne). Aux États-Unis, l'ouverture d'écoles polonaises fut et reste facilitée par le fait que l'enseignement y est en majorité d'ordre privé.

6 Pourquoi en France, les immigrés n'agiraient-ils pas de même? Une France amie qui vient de contribuer à la renaissance de leur État, la France qui par la bouche de Clemenceau a plaidé à la Conférence de la Paix pour une Pologne spacieuse contrairement aux vœux de Lloyd George qui aurait préféré en limiter la frontière orientale à la Ligne Curzon?

7 Le traité de Versailles à peine conclu, Paris et Varsovie entament des négociations en vue d'une convention d'émigration/immigration. La France pleure ses morts $(1,4$ million d'hommes), panse ses blessés, ses amputés, ses gazés inaptes au travail, fait l'inventaire des villes détruites, des champs à déminer, des puits de charbon inondés par l'ennemi avant sa retraite. La France a besoin de bras tandis que la Pologne souffre de surpeuplement rural: des myriades de paysans sans terre peinent à s'employer comme ouvriers agricoles, ou bien possèdent des lopins minuscules, insuffisants pour nourrir tous les membres de la famille. Une immense misère affecte les campagnes polonaises.

8 La Convention, signée le 3 septembre 1919, donne le coup d'envoi à une immigration d'un type nouveau, à la fois collective et organisée. Les partants, sélectionnés au préalable en fonction de leurs aptitudes physiques (robustesse, mains calleuses de préférence - on n'a pas besoin d'intellectuels) signent un contrat d'un an qui leur garantit un emploi, un logement, l'égalité des salaires avec les Français de même qualification. Ce contrat va leur permettre de gagner leur pain et, ils l'espèrent, de mettre un peu d'argent de côté afin d'acheter au retour les quelques ares nécessaires pour assurer l'avenir. 
9 Mais les contrats, centrés sur l'emploi du migrant, restent muets sur le sort des épouses et des enfants qui accompagnent ou rejoindront le signataire. Dans l'un et l'autre camp règne l'idée que cette immigration ne durera pas, que la France une fois reconstruite saura se passer des travailleurs étrangers et que la Pologne pourra bientôt fournir un emploi décent à tous ses citoyens. La Convention prévoit néanmoins des réunions annuelles bilatérales, se déroulant soit à Paris, soit à Varsovie, afin de régler les questions restées en suspens. Parmi elles, la question scolaire. Celle-ci se décline en deux points: l'obligation de fréquenter l'école primaire concerne-t-elle les enfants d'immigrés? Et faut-il prévoir un enseignement spécifique dans leur langue maternelle pour les petits Polonais?

10 Si l'on reprend les termes de la loi Ferry du 28 mars 1882, on s'aperçoit de leur ambiguïté. L'article 4 affirme que "l'instruction primaire est obligatoire pour les enfants des deux sexes de six à treize ans révolus ». Le fait de ne pas mentionner de nationalité semble plaider en faveur d'une interprétation large: tous les jeunes, français ou non, y seraient astreints. Pourtant, en 1933, un projet de loi adopté par le Sénat précisera que «l'obligation scolaire s'applique aux enfants français et aux enfants étrangers ayant leur résidence en France ». Et la loi du 9 août 1936 - une des premières mesures du gouvernement de Front Populaire - mettra fin à toute interprétation négative en affirmant «l'instruction primaire obligatoire pour les enfants des deux sexes, français et étrangers, de six ans à quatorze ans révolus ».

11 Pourquoi faut-il le dire avec une telle clarté ? Parce que, en cas d'afflux soudain de petits étrangers en grand nombre, le directeur ou la directrice d'école pouvait auparavant refuser de les accueillir. C'est ce qui s'est produit, à plusieurs reprises dans les cités minières au début des années vingt à l'égard de Polonais. L'Italie, la première, avait dans un accord signé en 1910, obtenu que l'obligation scolaire s'appliquât aux enfants de ses ressortissants travaillant en France. La Pologne insiste à son tour et, en avril 1924, lors d'une entrevue entre François Sokal, ministre polonais de l'Instruction publique et les autorités françaises, ce point se règle facilement. Désormais, même en cas d'afflux, tous les jeunes Polonais iront à l'école: aucun refus d'inscription ne sera toléré. La question est capitale pour la République de Pologne qui veut mettre fin à l'analphabétisme d'une partie de sa population. D'ailleurs, une des premières lois adoptées par la Diète de Varsovie aussitôt après la proclamation de l'indépendance (11 novembre 1918) fut d'instaurer un enseignement primaire obligatoire pour tous.

12 Le deuxième point est beaucoup plus épineux. Ce que demandent les autorités polonaises va à l'encontre de la législation française, laquelle cherche à lutter contre les dialectes régionaux et ne peut en même temps admettre des cours en langue étrangère. Après de longues tergiversations, année après année, et faute de pouvoir enfreindre ouvertement la loi, l'État finit par se défausser sur les entreprises privées, en particulier sur le Comité central des Houillères de France (CCHF), plus gros demandeur de main-d'œuvre étrangère. L'accord Sokal-Peyerimhoff, du nom du ministre polonais qui préside la réunion et du vice-président du CCHF, signé en avril 1924, n'a jamais paru au Journal officiel. Cet accord se présente sous la forme d'une lettre du second au premier.

Monsieur le Président,

À la suite des entretiens que nous avons eu l'honneur d'avoir avec vous et les membres de votre Délégation, et eu égard aux fonctions matérielles et au rôle moral dévolus à la Délégation des employeurs français en Pologne, commune au Comité Central des Houillères de France et à la Confédération Agricole des Régions 
Dévastées, nous avons satisfaction à vous marquer la communauté de vues qui s'est dégagée de nos conversations au sujet de l'enseignement des enfants polonais en France.

Cette question soulève de légitimes préoccupations, dont nous entendons ne pas nous désintéresser, et à l'apaisement desquelles nous apportons volontiers notre concours.

Nous nous déclarons donc prêts à inviter, de la manière la plus pressante, les employeurs, ressortissants à nos groupements, à inaugurer et à poursuivre la réalisation du programme visant, dans le cadre et dans les délais variables selon les professions, les régions et les moyens, à l'institution de l'enseignement ci-après défini :

a/ soit, en ce qui concerne les écoles publiques, en supportant les frais de l'enseignement complémentaire qui pourrait être institué, de la langue, de l'histoire et de la géographie polonaises ;

b/ soit, lorsque l'effectif des enfants polonais le justifiera, c'est-à-dire plus généralement dans la grande industrie et, en particulier, à plus brève échéance, dans l'industrie houillère, à raison de l'importance et de la densité de ses effectifs polonais, en ouvrant, au fur et à mesure des besoins, des écoles privées, où sera assuré, sous la surveillance des autorités académiques françaises, et dans le cadre du programme scolaire normal, le même enseignement, complété, s'il y a lieu, pour les jeunes enfants qui n'auraient pas encore de notions suffisantes de la langue française, par l'enseignement d'autres matières en polonais.

Les candidats destinés à assurer la part polonaise de l'enseignement ci-dessus prévu seront présentés par l'Administration polonaise (ministère de l'Instruction publique et des Cultes) à l'agrément d'une Commission privée d'examen qui recommandera aux employeurs pour engagement les candidats reconnus par elle pourvus des aptitudes pédagogiques nécessaires.

Nous considérons que la présence dans une exploitation d'un nombre d'enfants polonais d'âge scolaire, supérieur à 65 , devra appeler, de notre part l'invitation pressante, à laquelle il est fait allusion plus haut, tendant à l'engagement par les soins de cette exploitation de personnel enseignant polonais.

Le traitement de ce personnel sera fixé d'après les mêmes règles que pour les instituteurs et institutrices français des mêmes écoles privées.

Veuillez agréer, Monsieur le Président, les assurances de notre haute considération.

Le vice-président

Signé : Peyerimhoff ${ }^{4}$

L'auteur de la lettre promet d'« inviter » les employeurs à organiser des cours pour les fils et filles de leurs ouvriers. Il n'affirme pas qu'ils le feront. Mais la faveur consentie pour les petits Polonais par rapport aux autres enfants étrangers est considérable.

14 L'État intervient à la marge. Le 30 juin 1924, le ministère français de l'Instruction publique transmet aux établissements primaires une circulaire indiquant la possibilité d'accueillir des moniteurs étrangers qui enseigneraient leur propre langue aux élèves de même origine, le soir après les six heures de classe. Le 21 décembre 1925, une deuxième circulaire rappelle la distinction entre le régime des écoles libres et les autres. Officiellement, il n'est pas question d'un privilège fait aux seuls Polonais. Si les immigrés italiens paraissent peu intéressés par cette mesure, c'est elle qui va permettre à des ressortissants tchécoslovaques d'ouvrir eux aussi des cours pour leurs enfants, infiniment moins nombreux que les petits Polonais (voir la communication de JeanPhilippe Namont). 


\section{Le mythe de «l'École polonaise »}

15 Les intéressés ont parfois anticipé, créant les premiers cours avant l'accord de 1924, tant la chose leur paraissait aller de soi. C'est le cas à Freyming (Moselle) au printemps 1921 où les leçons ont lieu dans un café, à Knutange (Moselle également) en 1922, à Rosières (Cher) en 1923 où arrive une enseignante de Pologne qui s'occupe alternativement des garçons et des filles. La volonté d'un enseignement en langue polonaise fédère tous les immigrés, y compris ceux qui furent scolarisés en allemand dans la zone prussienne, peut-être précisément à cause de la germanisation forcée qu'ils eurent à subir.

16 Les cours polonais vont surtout se développer en secteur minier : Pas-de-Calais et Nord charbonniers, Lorraine $\mathrm{du}$ fer, Lorraine orientale houillère, Alsace potassique, Normandie ferrifère, bassin de la Loire, autrement dit là où la présence polonaise est la plus forte. Il ne s'agit ni d'écoles proprement polonaises, ni même de classes polonaises, mais de cours ajoutés à l'horaire normal dans les établissements publics (le soir, voire le jeudi) ou insérés à l'intérieur de l'emploi du temps dans les écoles privées des Houillères qui ont la préférence des parents, soucieux de cet enseignement complémentaire. Les enseignants, simples moniteurs ou monitrices, placés sous l'autorité de l'instituteur français, salariés par l'entreprise ou par le consulat de Pologne, exercent dans des conditions précaires, dispensant quelques heures par semaine devant chaque groupe d'enfants et se partageant souvent entre plusieurs établissements, parfois entre deux ou trois cités voisines. L'expression «École polonaise " (Szkoła Polska), couramment employée pour désigner ces enseignements complémentaires, constitue un abus de langage et peut prêter à confusion.

17 Même dans les communes les plus densément polonaises, l'expérience aboutit à des résultats extrêmement variables. D'abord, par manque de moniteurs, car la Pologne donne la priorité à la formation d'enseignants affectés sur son propre territoire. Quand un moniteur quitte son poste et n'est pas remplacé, ou quand son successeur tarde à venir, c'est l'interprète de la compagnie ou un employé des bureaux qui fait cours, voire un parent d'élève ou tout autre bénévole. Le niveau est donc très inégal, parfois faible. D'autre part, le directeur d'école, souvent hostile, en tous cas indifférent, en dispense les élèves de la classe du certificat d'études. D'ailleurs quelques heures par semaine ne suffisent pas pour corriger les erreurs transmises par les parents au parler dialectal. Les jeunes Polonais élevés en France et rentrés en Pologne après 1945 s'apercevront vite de leurs insuffisances et devront s'inscrire à des cours de rattrapage.

L'enseignement en langue polonaise ne progresse que lentement. Une statistique pour l'année 1926, établie par les soins de l'Union des enseignants de polonais, tend à prouver que seuls 9715 sur les 34460 enfants d'âge scolaire profitent de leurs cours, soit à peine plus d'un quart. Si à Montceau-les-Mines, ce chiffre s'élève à 1040 sur 1200 , par contre à Carmaux près de 1000 élèves sont totalement privés de cette formation faute de maitre. Le Nord et le Pas-de-Calais, où pourtant les mineurs vivent groupés en colonies très denses, n'atteignent même pas la moyenne nationale avec 6630 bénéficiaires sur 24890 jeunes de six à treize ans. On ne compte encore que 88 moniteurs et monitrices dans toute la France dont 51 dans le bassin du Nord, soit respectivement un pour 110 et 130 enfants. L'Union fixe à 271 , dont 181 dans le NordPas-de-Calais, les effectifs nécessaires pour permettre un enseignement valable dans les 
établissements des Houillères et assurer un encadrement des élèves des écoles communales à l'heure de l'étude du soir.

À l'origine destinés aux «jeunes enfants qui n'auraient pas encore de notions suffisantes de la langue française » comme l'écrivait Henri de Peyerimhoff, les cours de polonais auraient dû, peu à peu, s'effacer. C'est le contraire qui se produit. Ils vont perdurer pendant près d'un demi-siècle et même augmenter en nombre jusqu'aux années 1950. Des inspecteurs venus de Pologne parcourent les cités polonaises, entrent dans les classes et écoutent les leçons comme le ferait n'importe lequel de leurs collègues œuvrant sur son territoire national. Grâce aux rapports qu'ils adressent à leur hiérarchie, nous possédons, à des dates données, des chiffrages extrêmement précis sur le type d'école (privée ou communale), le nombre d'élèves inscrits, les horaires pratiqués et les niveaux d'enseignement. Par exemple, au premier trimestre de l'année scolaire 1937-1938 (octobre à décembre), en la localité dénommée Méricourt Corons (compagnie des mines de Courrières), à l'école des Houillères pour garçons, 114 élèves suivent l'enseignement en polonais à raison de $7 \mathrm{~h} 30$ hebdomadaires réparties sur trois niveaux ; à l'école des Houillères pour filles, 117 élèves ont droit au même horaire, mais réparti sur quatre niveaux. Autre exemple, à Houdain (compagnie des mines de Bruay), l'école communale mixte compte 4 heures hebdomadaires réparties sur quatre niveaux et ceci pour 300 élèves, soit seulement une heure par groupe à raison d'une moyenne de 75 élèves chacun. Au total, selon ce rapport détaillé, l'inspecteur a visité 44 établissements dans le ressort du consulat polonais de Lille qui couvre les deux départements du Nord et du Pas-de-Calais. Il signale à chaque fois la présence ou non d'un autre enseignant sur place et constate que les moniteurs sont rarement seuls lorsqu'ils exercent dans une école privée des Houillères; l'instituteur français est là aussi. Par manque de locaux, tous deux se partagent les élèves, l'un donnant des exercices écrits pendant que l'autre fait cours à haute voix, ce qui évoque les écoles de villages à classe unique. Par contre, à la " communale ", il arrive que le moniteur (ou la monitrice) polonais(e) soit effectivement seul(e). Et pour cause. Les leçons ont lieu après la sortie du soir et les petits Français sont rentrés chez eux ${ }^{5}$.

Un autre rapport établi à la date du $1^{\text {er }}$ avril 1939 et concernant la France entière donne une vision d'ensemble de la situation à la veille du conflit mondial. Il y est fait état de :

1. 117 cours de polonais dans le ressort du consulat polonais de Lille (à Frais-Marais, Pecquencourt, Waziers, Sin-le-Noble, Bruay-en-Artois, Haillicourt, Houdain, Divion, Harnes, Méricourt, Sallaumines, Leforest, Dourges, Lens, Liévin, Avion, Barlin, Marles-les-Mines, Noeux-les-Mines, Ostricourt, Libercourt, etc.)

2. 53 dans celui de Strasbourg (à Creutzwald, Hayange, Hagondange, Uckange, Wittelsheim, Wittenheim, Stiring-Wendel, Freyming, Saint-Avold, Thionville, Ronchamp, etc.)

3. 42 dans celui de Lyon (Roche-la-Molière, Blanzy, Montceau-les-Mines, Le Creusot, Montchanin, Saint-Eloy-les-Mines, Beaulieu, Firminy, Les Gautherets, etc.)

4. 27 dans celui de Paris (Potigny, Rosières, Drancy, Paris, Blanc-Mesnil, Revin, etc.)

5.7 dans celui de Marseille (Alès, Le Martinet...)

6. 3 dans celui de Toulouse (Carmaux, Cransac, Cagnac-les-Mines).

21 Soit un total de 249 points concernées à raison, souvent, de plusieurs établissements par localité, ne serait-ce que du fait de la séparation entre une école pour garçons, et une pour les filles, entre deux écoles publiques et autant d'écoles privées, entre plusieurs quartiers possédant chacun leurs établissements scolaires ${ }^{6}$. On n'est pas loin alors de l'objectif fixé en 1926 par l'Union des enseignants polonais. Mais répétons-le : 
le nombre de cours ne garantit ni un suivi horaire suffisant, ni des résultats satisfaisants.

Par contre, cette formation complémentaire joue un rôle essentiel sur le plan culturel. Elle donne des éléments d'histoire de la Pologne, dont le petit Lavisse ne dit mot. Elle permet aux enfants d'honorer le pays de leurs aïeux, avec ses neiges, ses bouleaux, ses saules, ses récits légendaires, sa littérature, de réciter quelques vers d'Adam Mickiewicz, le Victor Hugo de la Pologne.

Si cet enseignement, initialement prévu par le camp français comme temporaire, s'est au contraire développé, c'est évidemment dû aux pressions des autorités de Varsovie, soucieuses de ne pas perdre contact avec les Polonais émigrés. Le nombre de moniteurs envoyés en France augmente au fur et à mesure que les besoins sont assurés sur le territoire même de la Pologne. En 1926, on en comptait 88, en 1939 ils sont 127 et, après la Libération, 287. Car les immigrés qui refusent de rentrer dans un pays devenu communiste, espèrent et attendent la chute de ce régime pour pouvoir retourner chez eux : plus que jamais, il leur semble essentiel de maintenir le lien linguistique. Dans les années 1970-80, il existe encore des cours de polonais là où vivent des volontaires pour les assurer. C'est le cas à Stiring-Wendel (Moselle), à Potigny (Calvados) grâce au couple Ginter, lui d'abord dans l'entre-deux-guerres, lui et sa femme ensuite, puis elle seule après son veuvage. Et bien entendu, dans plusieurs cités minières du Pas-de-Calais et du Nord. Mais, comme le disait très justement Władysław Ginter : « Je suis passé peu à peu d'un enseignement en polonais à l'apprentissage du polonais $»^{7}$. Car les petits enfants d'immigrés (troisième génération) ne parlent plus chez eux la langue du pays d'origine.

L'enseignement complémentaire donné en polonais à leurs parents, puis éventuellement à eux-mêmes n'a donc pas nui à la progressive intégration des descendants du demi-million de Polonais immigrés en France pendant l'entre-deuxguerres. Il faut chercher ailleurs les causes des difficultés rencontrées à l'heure actuelle par certains des enfants de migrants récents.

\section{BIBLIOGRAPHIE}

FREY, Yves (2003). Polonais d'Alsace. Pratiques patronales et mineurs polonais dans le bassin potassique de Haute Alsace, 1918-1948, Besançon, Presses universitaires franc-comtoises.

GOGOLEWSKI, Edmond (1994). La langue polonaise dans l'enseignement élémentaire et secondaire en France (1833-1990), Lille, Centre d'étude de la culture polonaise de l'université Charles de GaulleLille III.

PONTY, Janine (1977). « Les problèmes soulevés par la scolarisation des enfants polonais en France après la Première Guerre mondiale », Relations Internationales, $n^{\circ} 12$, p. 327-349.

- (1988). Polonais méconnus. Histoire des travailleurs immigrés en France dans l'entre-deux-guerres, Paris, Publications de la Sorbonne, (réédition en 2005). 
- (1995). Les Polonais du Nord ou la mémoire des corons, Paris, Autrement, (dernière réédition en 2008).

- (2004). L'immigration dans les textes, France 1789-2002, Paris, Belin.

- (2008). Les Polonais en France, de Louis 15 à nos jours, Paris, Editions du Rocher.

PONTY, Janine (sous la direction de) (2011). Polonia. Des Polonais en France de 1830 à nos jours, catalogue de l'exposition « Polonia », Paris, CNHI/Montag.

POTTIER, Marc (1999). Normands de tous pays. L'immigration étrangère en Basse Normandie de 1900 à 1950, Cabourg, Cahiers du Temps.

\section{NOTES}

1. Relations Internationales, $1977, \mathrm{n}^{\circ} 12, \mathrm{p} .327-349$.

2. Janine Ponty, Polonais méconnus. Histoire des travailleurs immigrés en France dans l'entre-deuxguerres, Paris, Publications de la Sorbonne, 1988 (réédition 2005), p. 352-353.

3. William Thomas and Florian Znaniecki, The Polish Peasant in Europe and America, Chicago, 1919.

4. Janine Ponty, L'immigration dans les textes, Paris, Belin, 2004, document $n^{\circ}$ 94, p. 173-175.

5. Archives des Actes Nouveaux (AAN), Varsovie, MSZ 10658.

6. Ibid., MSZ 10671.

7. Interview réalisée par Anna Masiewicz dans le cadre de ses travaux sur les modifications linguistiques du polonais en terre migratoire, recherches s'appuyant sur l'exemple de Potigny (Calvados).

\section{RÉSUMÉS}

Les ouvriers polonais recrutés sur contrat pour travailler en France dans l'entre-deux-guerres ont demandé et obtenu en 1924 qu'une dérogation fût apportée aux lois de Jules Ferry, afin de permettre à leurs enfants de recevoir un enseignement complémentaire en langue maternelle. Les Polonais sont donc à l'origine des arrêtés ministériels de 1925 et de 1929 qui ont ouvert la possibilité d'embaucher des "moniteurs scolaires étrangers " à l'intention des fils et des filles d'immigrés. Les cours donnés en polonais n'ont pas nui à la progressive intégration des descendants du demi-million de Polonais venus en France. Il faut chercher ailleurs les causes des difficultés rencontrées à l'heure actuelle par certains des enfants de migrants récents.

Recruited on a contract basis to work in France between the two world wars, workers from Poland asked in 1924 for an exemption from the laws enacted by Jules Ferry, and were granted the right for their children to be given a complementary education in their mother tongue. The Poles themselves are therefore at the origin of the ministerial orders of 1925 and 1929 that paved the way for the possible hiring of "foreign school tutors" in charge of the education of immigrants'daughters and sons, a scheme which did not hinder the progressive integration of the half-million descendants of Poles who came to France. The difficulties met today by some children of recent immigrants have other causes. 
INDEX

Mots-clés : Pologne, France, Polonais, immigration, travailleurs étrangers, enfants étrangers, scolarisation, élèves étrangers

Keywords : Poland, France, Polish, immigration, foreign labour, foreign children, schooling, foreign learners.

\section{AUTEUR}

\section{JANINE PONTY}

Professeur honoraire d'histoire contemporaine, université de Besançon

Commissaire scientifique de l'exposition « Polonia. Des Polonais en France depuis 1830 », Paris, CNHI/Palais de la Porte Dorée, mars-août 2011

Ponty.Janine@wanadoo.fr 